Carnets de géographes

GÉOGRAPHES.

$3 \mid 2011$

Les géographies des enfants et des jeunes

\title{
Les pratiques de loisirs des enfants et des jeunes en Ille-et-Vilaine : espaces et mobilités
}

\section{Olivier David}

\section{(2) OpenEdition \\ Journals}

Édition électronique

URL : http://journals.openedition.org/cdg/2114

DOI : $10.4000 /$ cdg. 2114

ISSN : 2107-7266

Éditeur

UMR 245 - CESSMA

Référence électronique

Olivier David, «Les pratiques de loisirs des enfants et des jeunes en Ille-et-Vilaine : espaces et mobilités », Carnets de géographes [En ligne], 3 | 2011, mis en ligne le 14 février 2019, consulté le 07 mai 2019. URL : http://journals.openedition.org/cdg/2114 ; DOI : 10.4000/cdg.2114

La revue Carnets de géographes est mise à disposition selon les termes de la Licence Creative Commons Attribution - Pas d'Utilisation Commerciale - Pas de Modification 4.0 International. 


\section{LES PRATIQUES DE LOISIRS DES ENFANTS ET DES JEUNES EN ILLE-ET-VILAINE : ESPACES ET MOBILITES}

OLIVIER DAVID

Université Rennes 2

UMR CNRS Espaces et Sociétés - ESO Rennes

Géographie

olivier.david@univ-rennes2.fr

\section{Résumé}

Les pratiques de loisirs dans le cadre du temps libre ne sont pas les mêmes pour tous les enfants et les jeunes, notamment en raison de leur lieu de résidence, de leurs caractéristiques individuelles et de leur environnement social et culturel. En posant l'espace comme cadre et support de l'action des sociétés, cet article cherche à mettre en lien les pratiques enfantines et juvéniles avec les caractéristiques des espaces de vie. L'espace s'inscrit inévitablement dans la praxis. Il est obligatoirement pris en compte par les acteurs (enfants, jeunes, parents, élus locaux, organisateurs d'activités, collectivités...), consciemment ou non, dans la mise en œuvre de leurs actions et de leurs stratégies. En décrivant les lieux de pratiques de loisirs, les mobilités afférentes, cette réflexion permet d'appréhender plus finement les modalités d'organisation de la vie quotidienne des familles, notamment par l'agencement subtil des temporalités et des territorialités de ses membres. 


\section{Introduction}

Les pratiques de loisirs des enfants et des jeunes s'inscrivent obligatoirement dans l'espace, en tant que support et cadre de l'action des individus et des groupes sociaux. De fait, les territoires vécus sont différenciés et dépendent simultanément des activités, des usages des lieux et des déplacements qui les accompagnent. Les territoires d'identité et d'action sont ainsi très variés et leur assemblage fonde la territorialité des acteurs. En ce qui concerne les enfants et les jeunes, l'espace de vie est structuré autour de plusieurs centralités différentes, parmi lesquelles on retrouve le domicile, l'établissement scolaire mais aussi les espaces de loisirs, les lieux de rencontres avec les pairs... A partir de cartes mentales réalisées par les jeunes eux-mêmes, certains auteurs ont même révélé " un espace topologique, une territorialisation discontinue qui distingue schématiquement trois types d'espaces : l'espace privé du domicile, son environnement proche (le village pour le périurbain, le quartier pour le citadin) et l'hypercentre de l'agglomération (qui représente l'environnement proche du domicile pour ceux qui y résident) " (Roméro, Dumont, 2008, p. 110). Les différents types d'espaces pratiqués et leur articulation constituent donc les territoires du quotidien des enfants et des jeunes.

Cette réflexion s'intéresse à la localisation des activités et des services fréquentés dans le cadre du temps libre ainsi qu'aux mobilités qu'ils impliquent, aussi bien chez les enfants et les jeunes que pour leurs parents, souvent contraints de les accompagner. Le temps " taxi » (accompagnement des enfants) représente en effet une part non négligeable du temps parental. Nos travaux montrent notamment que la mobilisation des parents est assez forte pour tout ce qui concerne l'accompagnement des enfants vers les activités et services socioéducatifs. Cette situation est en partie liée à la diversité des lieux de pratiques. Les pratiques de loisirs des enfants et des jeunes façonnent en retour les territoires du quotidien familial.

Cet article souhaite présenter la multiplicité des lieux de pratiques d'activités de loisirs ainsi que leur agencement. Si la mobilisation des ressources éducatives s'opère généralement dans une relative proximité par rapport au lieu de résidence, il n'est pas rare que certains jeunes pratiquent leurs activités dans un rayon d'action beaucoup plus étendu, coïncidant fréquemment avec le lieu de scolarisation. Il est donc particulièrement intéressant d'analyser les lieux de pratiques tout en les croisant avec les caractéristiques individuelles des enfants et des jeunes (âge, sexe, lieu de résidence et de scolarisation...). Les résultats révèlent d'importants effets de contexte. La dissociation entre le domicile et les lieux de pratiques implique des déplacements qui structurent fortement l'organisation familiale pour répondre aux besoins éducatifs et sociaux des enfants et des jeunes. Cette question des mobilités est très importante dans le quotidien familial et l'appréciation des familles sur les modalités d'organisation qu'elles génèrent est révélatrice de la place qu'elles occupent dans le temps parental. Ainsi, l'inscription spatiale des pratiques est lourde de conséquences dans le fonctionnement quotidien des ménages. Non seulement, elles interfèrent sur les choix d'activités et le recours aux services socio-éducatifs, mais elles peuvent parfois produire des contraintes sur la recherche et la mobilisation des ressources éducatives. 


\section{Les lieux de pratiques de loisirs des enfants et des jeunes}

La localisation et les mobilités engendrées par la pratique d'activités sont centrales dans l'organisation de la vie quotidienne des familles. Elles constituent une dimension essentielle dans l'appréhension de la territorialité des enfants et des jeunes. "Le rapport aux lieux n'existe donc pas en soi de façon indépendante, mais est toujours lié à la question des pratiques » (Stock, 2004).

Afin de décrire précisément les comportements et les pratiques de loisirs des enfants et des jeunes dans le cadre de leur temps libre, nous avons réalisé une enquête approfondie auprès des familles résidant en Ille-et-Vilaine . Le choix de ce département est lié à une opportunité de coopération scientifique avec le Conseil Général d'Ille-et-Vilaine qui a financé le déroulement de cette recherche.

\section{Appréhender les pratiques de loisirs des enfants et des jeunes}

En effet, la compréhension des pratiques sociales nécessitait une description exhaustive des activités socio-éducatives choisies par les enfants et les jeunes, c'est-à-dire l'ensemble des services mobilisés et des activités pratiquées dans le cadre du temps libre (hors temps scolaire, au sein et en dehors du cadre familial, organisés ou non). Ce champ englobe aussi bien les accueils de loisirs, les activités sportives et culturelles réalisées auprès d'associations ou de clubs, les écoles de musique, les activités ludiques et informelles, les sorties entre amis, les discussions sur internet... D'emblée, l'enquête a opté pour une vision globale des loisirs.

La diversité des facteurs explicatifs de ces pratiques nous a orientés vers plusieurs registres de questionnements, pour tenter de mettre en relation les choix des enfants et des jeunes avec les caractéristiques sociales et culturelles de leur environnement familial. Afin de mieux appréhender les effets d'âge et de genre, l'enquête s'est intéressée aux enfants âgés de 6 à 24 ans révolus. Ces bornes statistiques permettent de couvrir l'ensemble des niveaux scolaires (primaire, secondaire, supérieur...) mais aussi les problématiques spécifiques aux jeunes adultes vivant encore au domicile de leurs parents.

Dans la mesure où nous avons choisi de mesurer l'impact des contextes territoriaux sur la structure des pratiques d'activités et de services socio-éducatifs, il était nécessaire de construire une base de données originale, en croisant à la fois des variables spatiales (en fonction des lieux de résidence, des lieux de scolarisation, des lieux d'activité...), des variables socio-économiques (déterminants sociaux, socioprofessionnels...) et des variables culturelles (valeurs, représentations, perceptions...). Cette démarche avait l'ambition de décrire les pratiques de loisirs mais aussi de les analyser au filtre des caractéristiques de l'environnement social, culturel et territorial des enfants et des jeunes.

Le questionnaire a donc été construit autour de deux objets complémentaires, pour croiser, d'une part, les modes de vie des familles et des jeunes avec leurs pratiques spatiales en matière d'activités et de services de loisirs, et, d'autre part, appréhender et identifier les besoins des familles et des jeunes en matière de services de proximité ainsi que leurs appréciations sur les politiques éducatives mises en œuvre au plan local. Plusieurs questions ouvertes ont ainsi permis d'apprécier les comportements des familles, notamment lorsque l'activité ou les services fréquentés sont localisés en dehors de la commune de résidence. 
La constitution d'un échantillon aléatoire rigoureux s'appuyant sur une base de sondage exhaustive des familles était évidemment impossible à l'échelle d'un département comme celui de l'Ille-et-Vilaine. Nous avons donc réalisé un échantillon spatial, en respectant la méthode des quotas, définis en fonction de caractéristiques simples (âge, sexe, CSP...), et fidèles à la structure de la population départementale. La fiabilité de l'enquête impliquait une sélection de communes représentatives de la diversité des espaces urbains, périurbains et ruraux. Le choix des communes enquêtées a croisé les différents types du zonage en aires urbaines et en aires d'emplois de l'espace rural (ZAUER (C) INSEE), la desserte routière départementale et l'éloignement aux pôles urbains.

Enfin, la passation de l'enquête a été effectuée en plusieurs temps : une diffusion en porteà-porte des questionnaires pour garantir une sélection aléatoire des familles, un délai de deux semaines pour remplir le questionnaire en présence des enfants, une seconde visite dans les foyers pour compléter et recueillir les questionnaires renseignés. Des entretiens qualitatifs auprès d'une dizaine de familles ont complété le matériau de base.

\section{La diversité des lieux de pratiques}

D’un point de vue général, $74,4 \%$ des enfants pratiquent une activité ou fréquentent un service sur leur commune de résidence. C'est une première information qui traduit une relative proximité des services mobilisés dans le cadre du temps libre. Ils sont également $71,4 \%$ à pratiquer une activité sur leur commune de scolarité, la coïncidence avec celle du domicile étant très importante. Enfin, seulement $24,5 \%$ des enfants déclarent un autre lieu de pratique.

Les premiers résultats révèlent surtout l'importance du genre sur les pratiques de loisirs et par conséquent sur les lieux d'activités. Ainsi, la pratique hors commune de résidence est plus fréquente chez les filles. La nature des activités pratiquées semble expliquer cette caractéristique. En ce qui concerne les activités sportives et culturelles, la pratique des filles est davantage diversifiée que celle des garçons, et nécessite le recours à des services qui ne sont pas toujours accessibles sur le lieu de domicile.

L'exemple de la pratique sportive illustre très concrètement cette réalité. Le football, majoritairement pratiqué par les garçons, nécessite assez rarement l'inscription dans un club extérieur à la commune, sauf pour des motifs liés à la recherche d'un environnement de qualité dans le cadre de la pratique de haut niveau. Par contre, le choix de l'équitation, nettement plus élevé chez les filles, est inévitablement déterminé par les lieux d'activités, beaucoup moins répandus que les clubs de football. La fréquentation d'équipements hors commune de résidence est souvent incontournable. 
Figure 1 : Lieu de pratique des activités selon l'âge, le sexe et la commune de résidence

\begin{tabular}{|c|c|c|c|c|c|}
\hline Unité : \% & $\mathbf{6 - 1 0}$ ans & $\mathbf{1 1 - 1 7}$ ans & $\mathbf{1 8 - 2 4}$ ans & Garçons & Filles \\
\hline Commune de résidence & 78,4 & 72,2 & 69,4 & 77,1 & 71,7 \\
\hline Commune de scolarité & 76,9 & 67,5 & 67,5 & 68,9 & 74,0 \\
\hline Autre commune & 29,1 & 23,0 & 13,3 & 21,5 & 26,1 \\
\hline & \multicolumn{2}{|c|}{ Urbain } & \multicolumn{2}{c|}{ Périurbain } & \multicolumn{2}{c|}{ Rural } \\
\hline Commune de résidence & \multicolumn{2}{|c|}{91,4} & 58,9 & \\
\hline Commune de scolarité & 89,9 & 55,9 & \\
\hline Autre commune & \multicolumn{2}{|c|}{11,9} & 37,8 & 24,5 \\
\hline
\end{tabular}

Source: Enquête réalisée par l'auteur

Toutefois, si la nature des activités choisies définit fortement les lieux d'activité, la configuration de l'offre locale (sur les lieux de résidence des familles) est également déterminante dans les choix d'activités et de services socio-éducatifs. Les résultats de l'enquête soulignent en effet que les enfants vivant dans les communes périurbaines sont les plus fréquemment amenés à pratiquer leur activité en dehors de leur commune de résidence. Ils ne sont que $58,9 \%$ à fréquenter une structure locale, contre $91,4 \%$ des enfants vivant en ville ou $68,2 \%$ des enfants vivant en milieu rural. Ces différences sont évidemment liées à l'offre de services, beaucoup plus riche et diversifiée dans les villescentres des agglomérations qu'elle ne l'est sur les autres communes du département. Toutefois, il faut bien distinguer les pratiques périurbaines des pratiques rurales. Dans le premier cas, les familles n'hésitent pas à choisir un club sportif ou une activité sur les communes voisines, si la qualité et la nature des prestations fournies sont différentes. Il ne faut donc pas penser l'offre à l'échelle de la seule commune de résidence, mais dans un espace beaucoup plus vaste, plus proche du bassin de vie des familles périurbaines. En milieu rural, l'offre d'activités et de services est beaucoup moins riche et diversifiée. Les populations sont donc contraintes à choisir localement, d'autant plus que les communes voisines (ou dans un périmètre restreint) n'apportent pas davantage de choix. Enfin, les jeunes citadins sont beaucoup moins tentés d'aller chercher une activité extérieure, car l'offre locale permet de répondre à une bonne partie des besoins exprimés, sauf en cas d'activité très spécialisée.

Le niveau scolaire ne modifie que très marginalement les comportements des enfants et des jeunes. En revanche, la comparaison entre la commune de résidence et la commune de scolarité révèle d'importantes différences dans la territorialité du quotidien des enfants et des jeunes. Le changement d'établissement scolaire induit un élargissement spatial progressif des enfants et des jeunes dans leur vie quotidienne, avec une dissociation croissante entre lieu de résidence et lieu de scolarisation au fur et à mesure de l'âge des enfants, ou de la spécialisation de leur parcours de formation (notamment dans le cas des filières professionnelles et de l'apprentissage).

\section{La pratique hors lieu de résidence et ses motifs}

L'enquête a également permis d'étudier plus précisément les familles dont les enfants pratiquent une activité en dehors de leur commune de résidence. Les données ont permis 
de cartographier les aires de pratique, mais également de comprendre les motifs des parents.

Figure 2 : Lieux de pratiques pour les enfants scolarisés en élémentaire et dans le secondaire

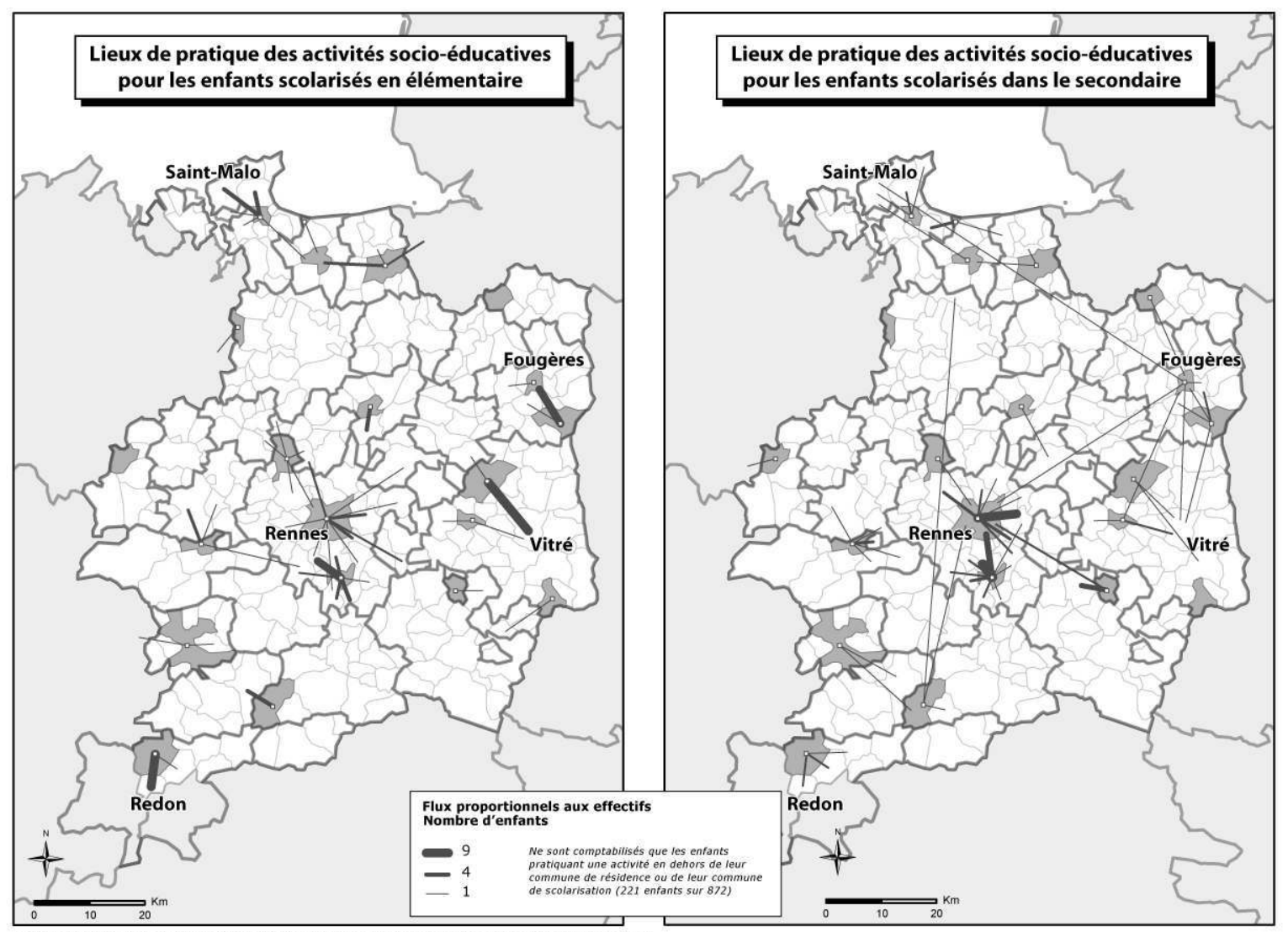

Source : Enquête réalisée auprès de 451 familles ayant des enfants âgés de 6 à 24 ans (juin-novembre 2006)
Conception : David O. - Réalisation : Lepetit A., UMR 6590 ESO

Les cartes réalisées à partir du recueil de données permettent d'appréhender plus concrètement les bassins de pratique des enfants et des jeunes, en fonction de leur commune de résidence. L'échantillon spatial de l'enquête ne permet de construire ce type de document qu'à partir des 21 communes sélectionnées. Les cartes produites montrent très distinctement un élargissement des aires de pratique en fonction de l'âge et du degré de scolarisation. Les enfants inscrits dans le primaire et qui pratiquent leur activité en dehors de leur commune de résidence le font dans un périmètre restreint, sur les communes limitrophes ou dans un rayon ne dépassant pas 20 kilomètres. Dans certains cas bien localisés, il est possible de remarquer la forte polarisation d'une commune urbaine. C'est le cas autour de Fougères, Vitré, Saint-Malo, Dol-de-Bretagne et Redon. Lorsque les enfants quittent l'école élémentaire pour le collège, ou ensuite pour le lycée, les lieux de pratiques des activités socio-éducatives se diversifient. Le réflexe de proximité s'impose toujours, puisque l'essentiel des communes cibles restent dans un périmètre restreint par rapport au domicile. Toutefois, les déplacements de plus grande distance augmentent très clairement, même si cela ne concerne que des effectifs limités. D'autre part, la diversification des lieux de pratique se traduit également par une moindre polarisation des villes-centres des agglomérations. 
En travaillant sur les motifs des parents en ce qui concerne la pratique extérieure à la commune de résidence, il a été nécessaire de classer les réponses en plusieurs catégories distinctes. La configuration de l'offre apparaît déterminante, puisque 63,4 \% des familles s'y réfèrent pour expliquer le choix d'une activité sur une autre commune. $C^{\prime}$ est une proportion très élevée qui montre bien l'impossibilité pour certaines familles de répondre aux souhaits de leurs enfants en matière d'activités et de services socio-éducatifs sur leur propre lieu de résidence. En contrepartie, cette réalité relègue au dernier plan la recherche de proximité, impossible devant de telles difficultés. Les motifs exprimant un choix délibéré des familles, ou relatifs à la qualité des services, ou bien répondant à des contraintes organisationnelles arrivent ensuite, représentant un peu plus d'un quart des réponses. Les contraintes liées à la scolarité se positionnent loin derrière avec 5,6 \% des motifs évoqués.

La répartition des réponses par catégorie de motifs en fonction du lieu de résidence souligne les contraintes spécifiques des communes périurbaines et rurales. L'insuffisance de l'offre est un motif exprimé approximativement par 2 familles sur 3, alors qu'en ville seulement $42,6 \%$ des ménages y font référence. Les possibilités de choix et de recherche de proximité sont plus faciles en ville qu'ailleurs, si l'on en juge par les réponses formulées par les parents. Les motifs liés à la recherche de qualité sont également plus importants en ville. Ce sont des différences très intéressantes sur l'appréciation des modes de vie des populations en fonction de leur commune de résidence. Les modalités de choix des services et activités et les contraintes sont bien différentes d'une commune à l'autre sur le département.

\section{Territorialités et mobilités associées aux loisirs}

Les résultats de l'enquête ont permis d'envisager un travail cartographique à plusieurs niveaux d'échelles. La constitution de la base de données offrait à la fois la possibilité de cartographier les pratiques au niveau de la cellule familiale mais aussi de consolider les résultats en fonction des types de communes de résidence (urbain, périurbain, rural). Cette production croisée apporte des renseignements très riches sur l'inscription spatiale des pratiques.

\section{Des pratiques différenciées selon les contextes territoriaux}

Pour travailler à l'échelle de la famille, nous avons été obligés de sélectionner plusieurs cas de figure, en cherchant à couvrir la diversité des configurations familiales, des contextes résidentiels et des mobilités associées aux pratiques d'activités dans le cadre du temps libre. Ce choix induit obligatoirement un biais dans l'analyse, mais nous avons cherché à être le plus exhaustif possible pour présenter l'hétérogénéité des situations. Les critères de sélection retenus ont donc été le nombre d'enfants, leur âge et leur sexe, les communes de scolarité, les communes d'activités de loisirs, les communes de travail des parents et le type de commune de résidence. La cartographie des différentes situations familiales décrit ainsi l'assemblage des territorialités des membres de la famille, en associant le lieu de résidence, les lieux de scolarité, les lieux de travail des parents et les lieux de pratiques d'activités des enfants et des jeunes. L'examen comparatif des différentes cartes permet de dégager quelques conclusions structurantes sur l'articulation des territorialités familiales. 
Figure 3 : Les territoires du quotidien familial

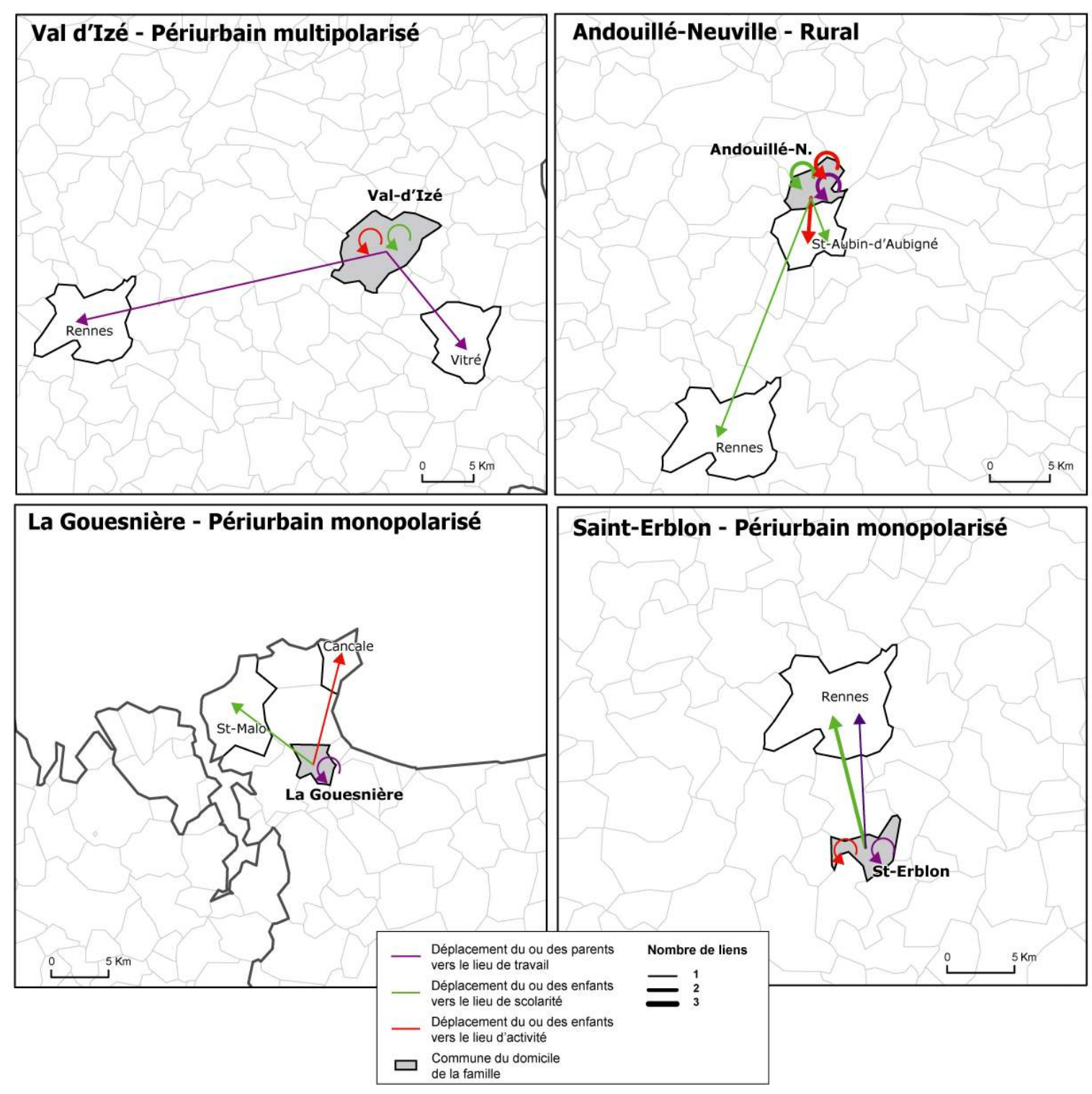

Lorsque l'on s'intéresse aux mobilités engendrées par la scolarité des enfants et l'activité professionnelle des parents, force est de constater que les pratiques spatiales familiales se complexifient. En milieu urbain, la coïncidence des lieux de scolarité, d'emploi et d'activités de loisirs est beaucoup plus forte qu'en milieu périurbain et rural. Par la richesse de l'offre éducative, scolaire et périscolaire, sportive et culturelle, mais aussi par la forte concentration des emplois, les familles vivant en ville ont des pratiques spatiales beaucoup plus simples, dans le sens où elles sont largement centrées sur leur commune de résidence. A l'échelle intra-urbaine, elles peuvent néanmoins engendrer des formes de mobilité complexes, ce que nous n'avons pas appréhendé. De plus, les enfants citadins sont généralement scolarisés à proximité du domicile, car le potentiel démographique autorise une plus forte densité d'équipements scolaires. 
Figure 4 : Les territoires du quotidien familial

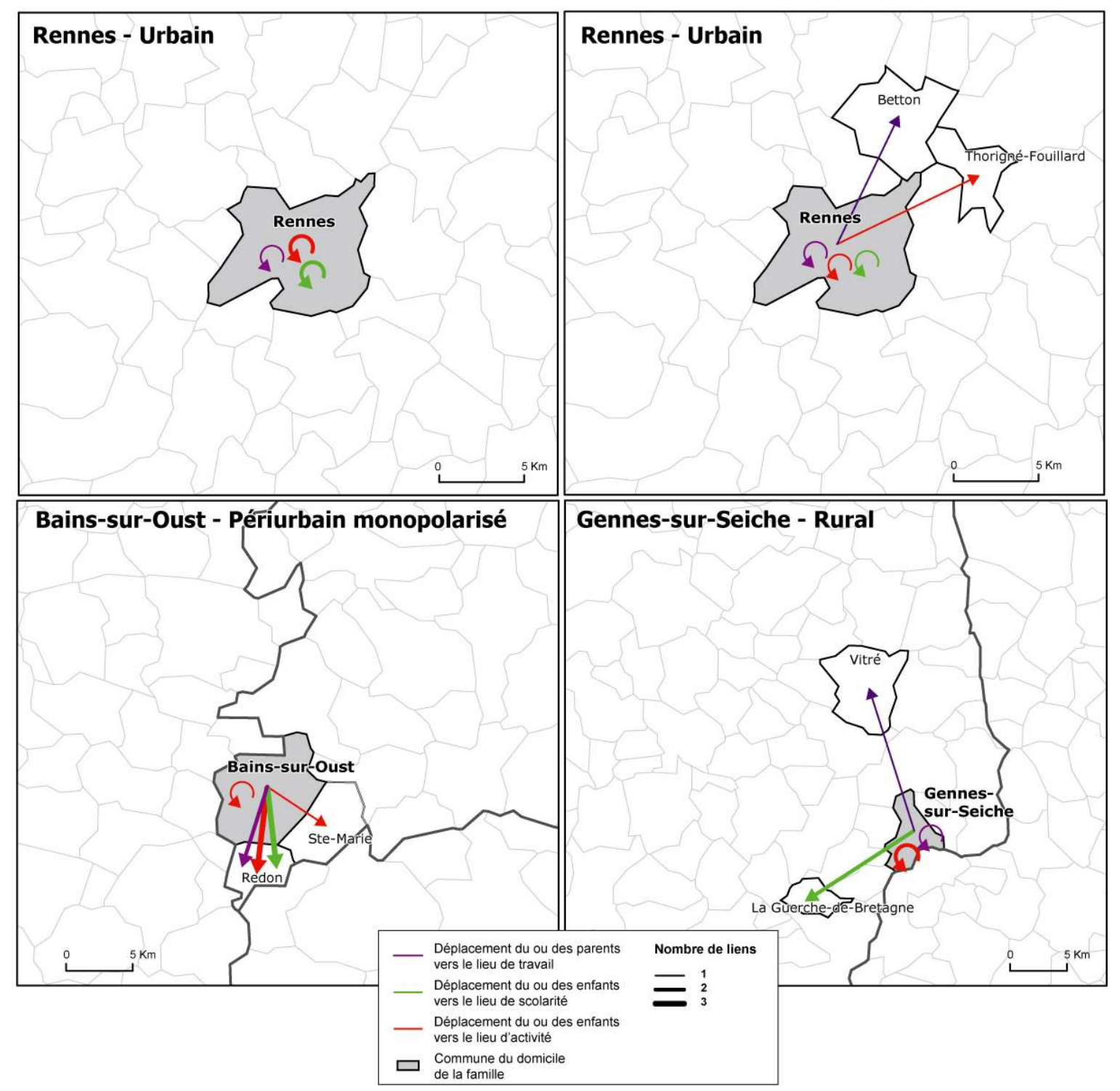

En revanche, dans les communes périurbaines et rurales, l'espace de vie familial est beaucoup plus éclaté. Les lieux d'activité professionnelle des parents sont souvent situés sur les pôles d'emploi les plus proches, parfois distincts d'un parent à l'autre. D'autre part, en fonction de l'âge des enfants, le passage au collège ou au lycée a la même conséquence sur les lieux de scolarisation. Cela contribue donc à façonner des territorialités individualisées complexes qui auront un véritable impact sur l'organisation quotidienne et hebdomadaire de la famille. Plusieurs exemples sélectionnés le démontrent, telle que la famille résidant à Andouillé-Neuville, commune rurale située au nord-est du pays de Rennes. Si les deux parents travaillent sur la commune, les deux enfants les plus âgés sont scolarisés sur SaintAubin-d'Aubigné (à $3 \mathrm{kms}$ ) et à Rennes (à $25 \mathrm{kms}$ environ). Les activités de loisirs nécessitent également un déplacement vers Saint-Aubin-d'Aubigné pour deux des quatre enfants. Les mobilités associées à ces différents lieux structurants de la vie quotidienne sont très contraignantes pour les individus mais également pour l'organisation collective de la cellule 
familiale. La famille domiciliée à La Gouesnière, composée d'un seul parent et d'un enfant, présente des modalités similaires. La mère travaille à proximité du domicile, mais l'enfant se déplace tous les jours sur Saint-Malo (à 7,5 kms) pour sa scolarité et à Cancale pour ses activités de loisirs. D'autres familles, vivant essentiellement en milieu rural et périurbain, sont dans des situations tout aussi compliquées.

Figure 5 : Contexte territorial et lieux de pratiques d'activités

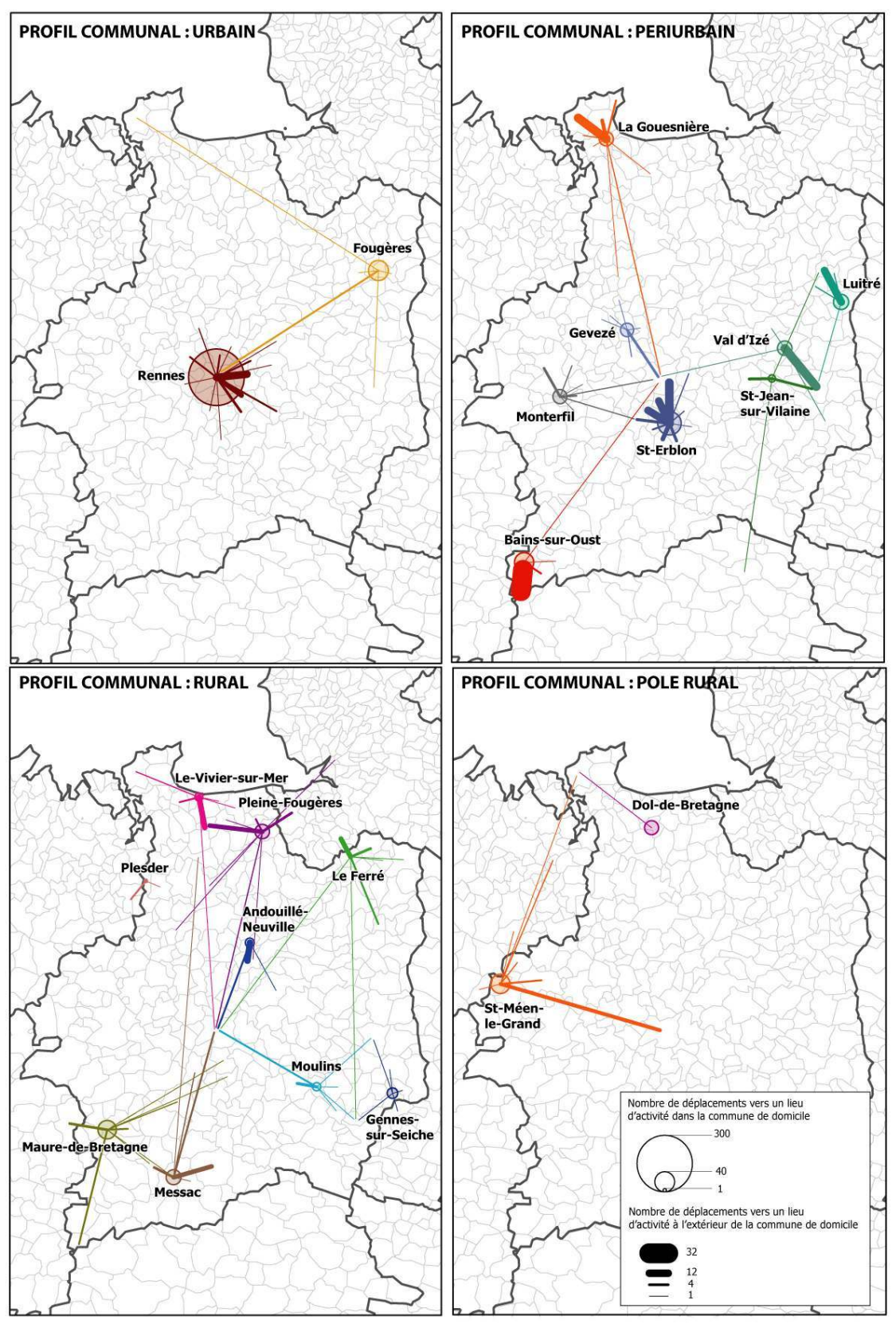

\section{Les mobilités associées aux pratiques de loisirs}

Les déplacements liés aux activités socio-éducatives sont apparus très importants dès les entretiens préalables à l'enquête. Tous les parents ont souligné le temps important consacré à l'accompagnement des enfants vers les structures d'accueil, ainsi que les difficultés à 
articuler les mobilités de chacun d'entre eux, en cas de fratrie. Le questionnaire a permis non seulement d'apprécier les modalités d'organisation, mais aussi les modes de déplacement retenus.

Figure 6 : Modalités de déplacement des enfants

\begin{tabular}{|c|c|c|c|c|}
\hline Unité : \% & $\begin{array}{c}\text { Déplacement } \\
\text { seul }\end{array}$ & $\begin{array}{c}\text { Accompagné par } \\
\text { un parent }\end{array}$ & $\begin{array}{c}\text { Accompagné par une } \\
\text { autre personne }\end{array}$ & Utilisation de transports combinés \\
\hline Ensemble & 51,0 & 67,9 & 18,5 & 6,0 \\
\hline $6-10$ ans & 22,8 & 90,2 & 23,3 & 1,8 \\
\hline $11-17$ ans & 61,4 & 64,4 & 17,1 & 7,0 \\
\hline $18-24$ ans & 94,3 & 19,5 & 10,6 & 5,8 \\
\hline Garçons & 52,9 & 65,7 & 20,9 & 6,2 \\
\hline Filles & 69,4 & 70,5 & 19,7 & 8,9 \\
\hline Urbain & 60,6 & 63,5 & 15,1 & 5,0 \\
\hline Périurbain & 41,9 & 73,7 & 22,6 & 0,0 \\
\hline Rural & 47,6 & 65,6 & 17,5 & Enquête réalisée par Olivier DAVID \\
\hline
\end{tabular}

Les résultats confirment la place centrale des parents dans l'accompagnement des déplacements liés aux activités socio-éducatives. Sur l'ensemble de l'échantillon, plus de deux enfants sur trois se déplacent le plus souvent avec leur père ou leur mère, alors qu'ils ne sont que $51 \%$ à le faire seuls. L'âge est la variable la plus influente. L'autonomie progressive des enfants s'accompagne ainsi par des déplacements seuls de plus en plus fréquents. S'ils ne sont que $22,8 \%$ entre 6 et 10 ans à se rendre seuls sur les lieux de pratique, ils sont plus de neuf sur dix entre 18 et 24 ans. Simultanément, l'accompagnement par un parent ou une autre personne diminue avec l'âge des enfants. Les différences de genre sont également sensibles, puisque les filles se déplacent plus souvent seules que les garçons.

Le croisement des résultats avec le lieu de résidence révèle une fois de plus la spécificité du milieu périurbain. Les enfants y sont beaucoup plus fréquemment accompagnés par leurs parents ou par une autre personne. A l'inverse, le milieu urbain semble encourager davantage l'autonomie de déplacement des enfants, de même que l'utilisation de plusieurs modes de déplacement combinés. Dans ce cas, c'est souvent l'utilisation de transports collectifs qui est citée (métro, bus urbain...). En campagne, les déplacements seuls sont plus élevés qu'en milieu périurbain, mais une majorité d'enfants reste accompagnée par un parent.

Nous nous sommes ensuite intéressés aux modes de déplacements utilisés par les enfants se déplaçant seuls. Les comportements évoluent avec l'âge. Les plus jeunes se déplacent très majoritairement à pied $(67,2 \%)$ ou en vélo $(23,4 \%)$, alors que la motorisation ou le recours aux transports collectifs augmente progressivement avec l'âge. Les 11-17 ans se déplacent toujours majoritairement à pied $(35,4 \%)$ ou en vélo $(18,5 \%)$, mais utilisent également le bus $(38,1 \%)$ qui est le premier mode de transport de cette tranche d'âge. Scooter et cyclomoteur arrivent très loin derrière. Les jeunes de 18 ans et plus marquent leur différence par l'utilisation première de la voiture (34,5\%), tout juste devant le bus (33,3\%). Les déplacements non motorisés et individuels ne concernent plus que $20 \%$ des effectifs. 
Le lieu de résidence semble également avoir un impact sur les modalités de déplacements choisies par les enfants et les jeunes. La marche et le vélo sont très largement majoritaires dans les communes périurbaines et rurales, concernant plus de $60 \%$ des enfants, contre seulement $41,8 \%$ en ville. La différence s'explique par la moindre utilisation du vélo dans les communes urbaines, qui chute à $9 \%$ contre plus de $23 \%$ dans les autres communes. Les déplacements à pied sont moins fréquents en milieu urbain mais concernent tout de même près d'un enfant sur trois. En contrepartie, le bus est davantage sollicité en ville $(46,9 \%)$ que dans les autres communes. En ce qui concerne les déplacements motorisés, la proportion d'enfants utilisant un scooter ou un cyclomoteur est plus élevée dans les communes périurbaines et rurales (respectivement 9,5 et 8,5\%), alors qu'en ville elle n'intéresse que $2,3 \%$ des effectifs. Au même titre que le vélo, la plus grande dangerosité des conditions de circulation minimise sans aucun doute l'utilisation des deux roues. Le recours à la voiture est aussi plus fréquent en milieu rural et périurbain qu'en ville.

Le questionnaire a été construit pour mesurer, autant que faire se peut, les contraintes des familles en matière d'organisation des déplacements associés à la pratique d'activités socioéducatives. Les résultats globaux ne montrent pas d'insatisfaction majeure, puisque $66,3 \%$ des parents jugent facile l'organisation des déplacements. II convient cependant de noter d'importantes différences d'opinion selon le lieu de résidence. L'appréciation générale largement positive est assez nuancée, avec une part d'insatisfaits et de très insatisfaits beaucoup plus importante en milieu périurbain et rural qu'en milieu urbain. Ce constat nous renvoie aux caractéristiques de l'offre en milieu périurbain et rural, beaucoup moins structurée et moins diversifiée, obligeant les familles à recourir à des services extérieurs à leur commune de résidence, ce qui génère des mobilités beaucoup plus contraignantes qu'en ville. D'autre part, la moindre autonomie des enfants vivant en commune périurbaine ou rurale (par rapport à ceux résidant en ville) mobilise davantage les parents dans les déplacements vers les lieux d'activité. Ce " temps taxi » est très contraignant, notamment lorsque la taille des fratries est importante. Le croisement de cette variable avec la nature des réponses formulées par les parents montre en effet que le niveau d'insatisfaction des familles augmente avec le nombre moyen d'enfants.

Les motifs invoqués par les familles à propos de l'organisation des déplacements sont assez diversifiés. Plusieurs catégories ont été envisagées en fonction de la nature des réponses, afin de synthétiser l'information. Ces différentes réponses ont été croisées avec l'appréciation des parents sur l'organisation des transports. Les familles la jugeant très facile ont expliqué leur appréciation en soulignant principalement la proximité des activités $(55,8$ $\%$ ) et l'efficacité du système de transports collectifs (11,5\%). II convient de rappeler que ce sont des ménages vivant majoritairement dans des communes urbaines. A l'opposé, les parents exprimant des difficultés à organiser les déplacements évoquent essentiellement des problèmes d'articulation des temps des différents membres de la famille, en soulignant une conciliation difficile avec leurs horaires professionnels $(25,3 \%)$ et une gestion contraignante de l'emploi du temps de chaque enfant $(13,1 \%)$. Ils évoquent également la lourdeur des déplacements et la longueur des trajets (13,2 \%). Ces familles résident majoritairement sur des communes périurbaines et rurales, et affichent un nombre moyen d'enfants plus élevé que la moyenne de l'échantillon.

Bien évidemment, les pratiques et les mobilités de loisirs des enfants et des jeunes n'échappent pas à l'influence des caractéristiques sociales, qu'elles soient liées au genre ou 
à l'âge, mais aussi ou à la position socioprofessionnelle des parents. L'accessibilité financière ainsi que le coût des déplacements sont de véritables freins, fréquemment cités par les ouvriers et les employés. Le recours à des activités de loisirs adaptées aux attentes des enfants augmente progressivement avec le niveau de revenus. Les ressources culturelles et sociales des enfants et des jeunes sont déterminantes sur la nature et la qualité de leur temps libre, et viennent pondérer le seul poids de l'espace dans la structure des pratiques.

\section{Conclusion}

Cet article a permis d'appréhender l'inscription spatiale des pratiques d'activités socioéducatives des enfants et des jeunes dans le cadre de leur temps libre. Les résultats de l'enquête confirment les hypothèses formulées en introduction et montrent ainsi que la territorialité quotidienne des enfants et des jeunes est structurée par plusieurs lieux majeurs, au rang desquels figurent le domicile, l'école ou l'établissement de scolarisation et les lieux de pratiques d'activités socio-éducatives. L'articulation des temporalités journalières s'opère ainsi dans un espace agencé autour de ces différents types de lieux.

En ce qui concerne notre objet de réflexion, les loisirs s'inscrivent très clairement dans une relative proximité par rapport au lieu de résidence. La pratique d'activités et le recours aux services socio-éducatifs se déploient dans un espace globalement restreint et proche des lieux de résidence ou de scolarisation des enfants et des jeunes. L'analyse détaillée des résultats permet néanmoins d'apprécier plusieurs niveaux d'échelles de pratiques. En effet, avec l'âge des enfants et la spécialisation des activités, l'espace des pratiques de loisirs s'élargit sensiblement. Ce processus est encouragé par l'éloignement de plus en plus important des établissements scolaires (collège, lycée) vis-à-vis du domicile. L'autonomie progressive des enfants et des jeunes, notamment en matière de mobilité, autorise ainsi la mobilisation de ressources éducatives dans un espace beaucoup plus vaste.

La configuration de l'offre locale de services et d'activités n'est pas étrangère à cet élargissement de l'espace des pratiques des enfants et des jeunes. Lorsqu'elle est réduite, comme dans les espaces ruraux et périurbains, elle motive la recherche d'activités sur d'autres communes que celle de résidence. Les citadins sont moins concernés par ces difficultés, dans la mesure où la complétude et la diversité de l'offre permettent de couvrir plus largement les besoins éducatifs et sociaux des familles. Néanmoins, lorsque les pratiques en dehors de la commune de résidence deviennent importantes, les lieux d'activités demeurent polarisés dans les centres de services urbains les plus proches (secteurs périurbains) ou dans les pôles ruraux (communes rurales).

Enfin, cette dissociation des lieux de résidence et de pratiques génère des mobilités importantes qui concernent toutes les tranches d'âge. Les résultats de l'enquête confirment le fort engagement des parents dans la prise en charge des déplacements des plus jeunes, mobilisant une part importante du temps parental (temps taxi). L'autonomie des plus âgés décharge en partie les parents de cette contrainte, sauf lorsque les activités sont éloignées et que les réseaux de transports en commun sont faiblement développés. Tous ces éléments créent un ensemble de contraintes supplémentaires dans l'organisation de la vie quotidienne des familles, dont le poids est différent d'un contexte territorial à l'autre. 


\section{Bibliographie}

CREPIN C., 2010, "Attentes d'encadrement et d'autonomie des adolescents à l'occasion des activités de loisirs ", Politiques sociales et familiales, Revue de la CNAF, n 99, pp. 121-129

DANIC I., DAVID O., DEPEAU S. (dir.), 2010, Enfants et jeunes dans les espaces du quotidien, Coll. Géographie sociale, PUR, 269 p.

DAVID O., 2007, "Vie familiale, vie professionnelle : une articulation sous tension ", Espace, Populations, Sociétés, n² 2007 2-3, pp. 191-202

DAVID O., 2010, Le temps libre des enfants et des jeunes à l'épreuve des contextes territoriaux, Habilitation à diriger des recherches, Université Rennes 2, Volume original, $328 p$.

GALLAND O., 2007, Sociologie de la jeunesse, Coll. U, Colin, 4ème édition, 248 p.

LESNARD L., 2009, La famille désarticulée, Coll. Le lien social, PUF, 213 p.

OCTOBRE S., DETREZ C., MERCKLE P., BERTHOMIER N., 2010, L'enfance des loisirs. Trajectoires communes et parcours individuels de la fin de l'enfance à la grande adolescence, La Documentation française, $432 \mathrm{p}$.

OCTOBRE S., 2004, Les loisirs culturels des 6-14 ans, La documentation française, 429 p.

RAIBAUD Y., 2007, " Genre et loisirs des jeunes », Empan, n 65, pp. 67-74

ROMERO C., DUMONT M., 2008, "Les jeunes dans leurs mobilités. L'espace en tous sens ", in SECHET R., GARAT I., ZENEIDI D., 2008, Espaces en transactions, Rennes, PUR, coll. Géographie Sociale, pp. 109-119

STOCK M., 2004, "L'habiter comme pratique des lieux géographiques ", EspacesTemps.net, textuel, http://espacestemps.net/document 1138.html 